\title{
A Learning Approach to Name-Based Bug Detection
}

\author{
MICHAEL PRADEL, TU Darmstadt, Germany \\ KOUSHIK SEN, University of California, Berkeley, USA
}

Natural language elements in source code, e.g., the names of variables and functions, convey useful information. However, most existing bug detection tools ignore this information and therefore miss some classes of bugs. The few existing name-based bug detection approaches reason about names on a syntactic level and rely on manually designed and tuned algorithms to detect bugs. This paper presents DeepBugs, a learning approach to name-based bug detection, which reasons about names based on a semantic representation and which automatically learns bug detectors instead of manually writing them. We formulate bug detection as a binary classification problem and train a classifier that distinguishes correct from incorrect code. To address the challenge that effectively learning a bug detector requires examples of both correct and incorrect code, we create likely incorrect code examples from an existing corpus of code through simple code transformations. A novel insight learned from our work is that learning from artificially seeded bugs yields bug detectors that are effective at finding bugs in real-world code. We implement our idea into a framework for learning-based and name-based bug detection. Three bug detectors built on top of the framework detect accidentally swapped function arguments, incorrect binary operators, and incorrect operands in binary operations. Applying the approach to a corpus of 150,000 JavaScript files yields bug detectors that have a high accuracy (between $89 \%$ and 95\%), are very efficient (less than 20 milliseconds per analyzed file), and reveal 102 programming mistakes (with $68 \%$ true positive rate) in real-world code.

CCS Concepts: • Software and its engineering $\rightarrow$ Software notations and tools; Software verification and validation; $\bullet$ Computing methodologies $\rightarrow$ Neural networks;

Additional Key Words and Phrases: Bug detection, Natural language, Machine learning, Name-based program analysis, JavaScript

ACM Reference Format:

Michael Pradel and Koushik Sen. 2018. DeepBugs: A Learning Approach to Name-Based Bug Detection. Proc. ACM Program. Lang. 2, OOPSLA, Article 147 (November 2018), 25 pages. https://doi.org/10.1145/3276517

\section{INTRODUCTION}

Source code written by humans contains valuable natural language information, such as the identifier names of variables and functions. This information often conveys insights into the semantics intended by the developer, and therefore is crucial for human program understanding [Butler et al. 2010; Lawrie et al. 2006]. While the importance of identifier names for humans is widely recognized, program analyses typically ignore most or even all identifier names. For example, popular static analysis tools, such as Google Error Prone [Aftandilian et al. 2012] , FindBugs [Hovemeyer and Pugh 2004], or $\operatorname{lgtm}^{1}$, mostly ignore identifier names. As a result, analyzing a program that has

$\overline{1_{\text {https://gtm.com/ }}}$

Authors' addresses: Michael Pradel, TU Darmstadt, Department of Computer Science, Germany; Koushik Sen, University of California, Berkeley, EECS Department, USA.

This work is licensed under a Creative Commons Attribution 4.0 International License.

(C) 2018 Copyright held by the owner/author(s).

2475-1421/2018/11-ART147

https://doi.org/10.1145/3276517

Proc. ACM Program. Lang., Vol. 2, No. OOPSLA, Article 147. Publication date: November 2018. 
Table 1. Examples of name-related bugs detected by DeepBugs.

\begin{tabular}{|c|c|c|}
\hline ID & Buggy code & Description \\
\hline 1 & $\begin{array}{l}\text { browserSingleton. startPoller }(100 \\
\quad \text { function(delay, fn) } \\
\quad \text { setTimeout(delay, fn); } \\
\text { \}); }\end{array}$ & $\begin{array}{l}\text { The setTimeout function expects two } \\
\text { arguments: a callback function and } \\
\text { the number of milliseconds after } \\
\text { which to invoke the callback. The } \\
\text { code accidentally passes these argu- } \\
\text { ments in the inverse order. }\end{array}$ \\
\hline 2 & $\begin{array}{l}\text { for }(j=0 ; j<\text { param. replace } ; j++)\{ \\
\text { if }(\text { param.replace }[j] \text {. from }==\text { paramVal }) \\
\text { paramVal = param. replace }[j] . \text { to; } \\
\text { \} }\end{array}$ & $\begin{array}{l}\text { The header of the for-loop com- } \\
\text { pares the index variable } j \text { to } \\
\text { the array param. replace. Instead, } \\
\text { the code should compare } j \text { to } \\
\text { param. replace.length. }\end{array}$ \\
\hline 3 & 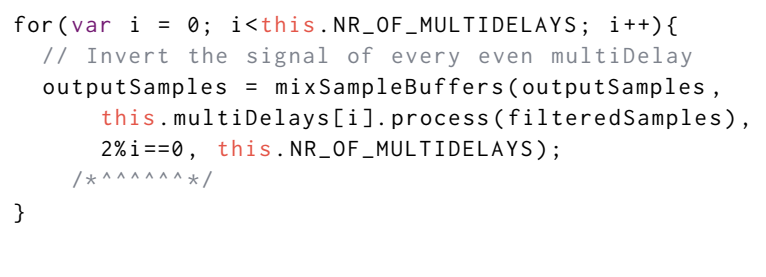 & $\begin{array}{l}\text { The highlighted expression } 2 \% i==0 \\
\text { is supposed to alternate between } \\
\text { true and false while traversing the } \\
\text { loop. However, the code accidentally } \\
\text { swapped the operands and should in- } \\
\text { stead be } i \% 2==0 \text {. }\end{array}$ \\
\hline
\end{tabular}

meaningful identifiers chosen by human developers yields the same results as analyzing a variant of the program where identifiers are consistently replaced with arbitrary and meaningless names.

Ignoring identifier names causes existing bug detection tools to miss bugs that, in hindsight, may appear obvious to a human. Table 1 gives three examples of such bugs. All three are from real-world code written in JavaScript, a language where identifiers are particularly important due to the lack of static types. Example 1 shows a bug in Angular.js where the developer accidentally passes two function arguments in the wrong order. The first expected argument is a callback function, but the second argument is called fn, an abbreviation for "function". Example 2 shows a bug in the Angular-UI-Router project where the developer compares two values of incompatible types with each other. In the absence of statically declared types, this inconsistency can be spotted based on the unusual combination of identifier names. Finally, Example 3 shows a bug in the DSP.js library where the developer accidentally swapped the operands of a binary operation inside a loop. A human might detect this bug knowing that $i$ is a common name for a loop variable, which suggests that the code does not match the intended semantics. As illustrated by these examples, identifier names convey valuable information that can help to detect otherwise missed programming mistakes.

One reason why most program analyses, including bug detection tools, ignore identifier names is that reasoning about them is hard. Specifically, there are two challenges for a name-based bug detector. First, a name-based analysis must reason about the meaning of identifier names. As a form of natural language information, identifier names are inherently fuzzy and elude the precise reasoning that is otherwise common in program analysis. Second, given an understanding of the meaning of identifier names, an analysis must decide whether a given piece of code is correct or incorrect. To be practical as a bug detection tool, the second challenge must be addressed in a way that yields a reasonably low number of false positives while detecting actual bugs. 
Previous work on name-based bug detection [Høst and Østvold 2009; Liu et al. 2016; Pradel and Gross 2011; Rice et al. 2017] addresses these challenges by lexically reasoning about identifiers and through manually designed algorithms. To reason about the meaning of identifiers, these approaches use lexical similarities of names as a proxy for semantic similarity. For example, the existing approaches may find length and len to be similar because they share a common substring, but miss the fact that length and count are semantically similar even though they are lexically different. To make decisions about programs, e.g., to report a piece of code as likely incorrect, existing name-based analyses rely on manually designed algorithms that use hard-coded patterns and carefully tuned heuristics. For example, a name-based analysis that has been recently deployed at Google [Rice et al. 2017] comes with various heuristics to increase the number of detected bugs and to decrease the number of false positives. Designing and fine-tuning such heuristics imposes a significant human effort that is difficult to reuse across different analyses and different classes of bugs.

This paper tackles the problem of name-based bug detection with a machine learning-based approach. To address the problem of reasoning about the meaning of identifiers, we use a learned vector representation of identifiers. This representation, called embeddings, preserves semantic similarities, such as the fact that length and count are similar. Such embeddings have been successful for several natural language processing tasks and adopting them to source code is a natural choice for name-based bug detection. To address the problem of deciding whether a piece of code is likely correct or incorrect, we formulate the problem as binary classification and train a model to distinguish correct from incorrect code. Because the classifier is learned without human intervention, the approach does not rely on designing and tuning heuristics.

Effectively learning a classifier that distinguishes correct from incorrect code requires training data that consists of both correct and incorrect examples. Examples of correct code are easily available due to the huge amounts of existing code, based on the common assumption that most parts of most code are correct. In contrast, large amounts of code examples that are incorrect for a specific reason are much harder to find. In particular, manually obtaining a sufficiently large data set would require a human to label thousands of bugs. To address this problem, we generate large amounts of training data via simple program transformations that insert likely bugs into existing, supposedly correct code. An important insight of our work is that learning from such artificially generated training data yields a learned model that is effective at identifying real-world bugs.

We implement our ideas into an extensible framework, called DeepBugs, that supports different classes of name-related bugs. The framework extracts positive training examples from a code corpus, applies a simple transformation to also create large amounts of negative training examples, trains a model to distinguish these two, and finally uses the trained model for identifying mistakes in previously unseen code. We present three bug detectors based on DeepBugs that find accidentally swapped function arguments, incorrect binary operators, and incorrect operands in binary operations. Creating a new bug detector consists of two simple steps. First, provide a training data generator that extracts correct and incorrect code examples from a given corpus of code. Second, map each code example into a vector that the machine learning model learns to classify as correct or incorrect. For the second step, all bug detectors reuse the same embedding of identifier names, simplifying the task of creating a name-based bug detector.

Our approach differs from existing bug detectors that identify bugs as anomalies in a corpus of code [Engler et al. 2001; Hangal and Lam 2002; Monperrus et al. 2010]. These approaches infer information from existing code by learning only from correct examples and then flag any code as unusual that deviates from the norm. To reduce false positives, those approaches typically filter the detected anomalies based on manually designed heuristics. Instead, our approach learns from positive and negative examples, enabling the machine learning model to accurately distinguish 
these two classes. DeepBugs differs from existing work on name-based bug detection [Høst and Østvold 2009; Liu et al. 2016; Pradel and Gross 2011; Rice et al. 2017] by reasoning about identifiers based on a semantic representation, by learning bug detectors instead of manually writing them, and by considering two new name-related bug patterns on top of the previously considered swapped function arguments [Liu et al. 2016; Pradel and Gross 2011; Rice et al. 2017]. Finally, our work is the first on name-based bug detection for dynamically typed languages, where name-related bugs may remain unnoticed due to the lack of static type checking.

We evaluate DeepBugs and its three instantiations by learning from a corpus of 100,000 JavaScript files and by searching mistakes in another 50,000 JavaScript files. In total, the corpus amounts to 68 million lines of code. We find that the learned bug detectors have an accuracy between $89 \%$ and $95 \%$, i.e., they are very effective at distinguishing correct from incorrect code. Manually inspecting a subset of the warnings reported by the bug detectors, we found 102 real-world bugs and code quality problems among 150 inspected. Even though we do not perform any manual tuning or filtering of warnings, the bug detectors have a reasonable precision of $68 \%$, i.e., the majority of the reported warnings point to actual bugs.

In summary, this paper contributes the following:

- A learning approach to name-based bug detection, which differs from previous name-based bug detectors (i) by reasoning about identifier names based on a semantic representation, (ii) by learning bug detectors instead of manually writing them, (iii) by considering additional bug patterns, and (iv) by targeting a dynamically typed programming language.

- We formulate bug detection as a classification problem and present a framework to learn a classifier from examples of correct and incorrect code. To obtain large amounts of training data for both classes, we create training data through simple program transformations that yield likely incorrect code.

- We implement the idea into a general framework that can be instantiated into different kinds of name-based bug detectors. The framework is available as open-source, enabling others to build on our work, e.g., by adding further bug detectors:

\section{https://github.com/michaelpradel/DeepBugs}

- We provide empirical evidence that the approach yields effective bug detectors that find various bugs in real-world JavaScript code.

\section{A FRAMEWORK FOR LEARNING TO FIND NAME-RELATED BUGS}

This section presents the DeepBugs framework for automatically creating name-based bug detectors via machine learning. The basic idea is to train a classifier to distinguish between code that is an instance of a name-related bug pattern and code that does not suffer from this bug pattern. By bug pattern, we informally mean a class of programming errors that are similar because they violate the same rule. For example, accidentally swapping the arguments passed to a function, calling the wrong API method, or using the wrong binary operator are bug patterns. Manually written bug checkers, such as FindBugs or Error Prone, are also based on bug patterns, each of which corresponds to a separately implemented analysis.

\subsection{Overview}

Given a corpus of code, creating and using a bug detector based on DeepBugs consists of several steps. Figure 1 illustrates the process with a simple example.

(1) Extract and generate training data from the corpus. This step statically extracts positive, i.e., likely correct, code examples from the given corpus and generates negative, i.e., likely incorrect, code examples. Because we assume that most code in the corpus is correct, the 


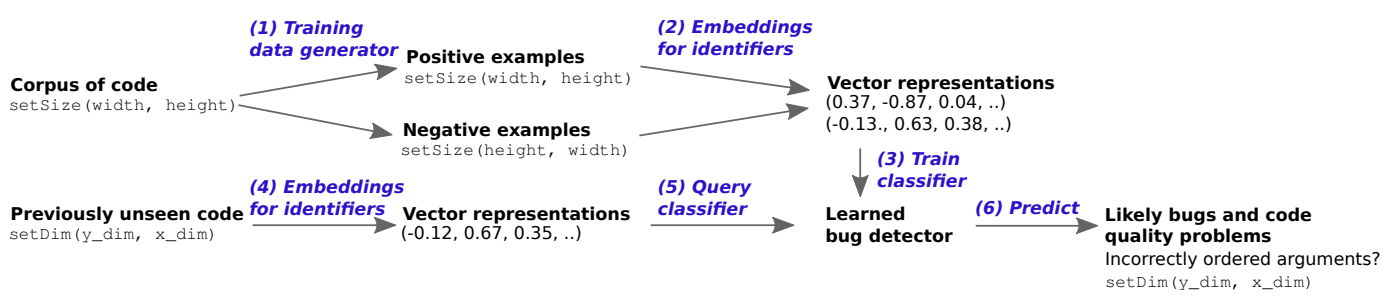

Fig. 1. Overview of our approach.

extracted positive code examples are likely to not suffer from the particular bug pattern. To also create negative training examples, DeepBugs applies simple code transformations that are likely to introduce a bug. (Step 1 in Figure 1.)

(2) Represent code as vectors. This step translates each code example into a vector. To preserve semantic information conveyed by identifier names, we learn an embedding that maps identifiers to a semantic vector representation via a Word2Vec neural network [Mikolov et al. 2013b]. (Step 2 in Figure 1.)

(3) Train a model to distinguish correct and incorrect examples. Given two sets of code that contain positive and negative examples, respectively, this step trains a classifier to distinguish between the two kinds of examples. The classifier is a feedforward neural network. (Step 3 in Figure 1.)

(4) Predict bugs in previously unseen code. This step applies the classifier obtained in the previous step to predict whether a previously unseen piece of code suffers from the bug pattern. If the learned model classifies the code to be likely incorrect, the approach reports a warning to the developer. (Steps 4 to 6 in Figure 1.)

The example in Figure 1 illustrates these steps for a bug detector aimed at finding incorrectly ordered function arguments. In Step 6, the bug detector warns about a likely bug where the arguments y_dim and x_dim should be swapped. The reason that the approach can spot such bugs is that the trained classifier generalizes beyond the training data based on the semantic representation of identifiers. For the example, the representation encodes that width and x_dim, as well as height and y_dim, are pairwise semantically similar, enabling DeepBugs to detect the bug.

\subsection{Generating Training Data}

An important prerequisite for any learning-based approach is a sufficiently large amount of training data. In this work, we formulate the problem of bug detection as a binary classification task and address it via supervised learning. To effectively address this task, our approach relies on training data for both classes, i.e., examples of both correct and incorrect code. As observed by others [Bielik et al. 2016; Nguyen and Nguyen 2015; Raychev et al. 2015], the huge amount of existing code provides ample of examples of likely correct code. In contrast, it is non-trivial to obtain many examples of code that suffers from a particular bug pattern. One possible approach is to manually or semi-automatically search code repositories and bug trackers for examples of bugs that match a given bug pattern. However, scaling this approach to thousands or even millions of examples, as required for advanced machine learning, is extremely difficult.

Instead of relying on manual effort for creating training data, this work generates training data fully automatically from a given corpus of code. The key idea is to apply a simple code transformation $\tau$ that transforms likely correct code extracted from the corpus into likely incorrect code. Section 3 presents implementations of $\tau$ that apply simple AST-based code transformations. 
Definition 2.1 (Training data generator). Let $C \subseteq L$ be a set of code in a programming language $L$. Given a piece of code $c \in C$, a training data generator $G: C \rightarrow\left(2^{C_{p o s}}, 2^{\left.C_{n e g}\right)}\right.$ creates two sets of code snippets $C_{\text {pos }} \subseteq C$ and $C_{n e g} \subseteq L$, which contain positive and negative training examples, respectively. The negative examples are created by applying transformation $\tau: C \rightarrow C$ to each positive example: $C_{n e g}=\left\{c_{n e g} \mid c_{n e g}=\tau\left(c_{p o s}\right) \forall c_{p o s} \in C_{p o s}\right\}$

By code snippet we mean a single expression, a single statement, or multiple related statements. Each code snippet contains enough information to determine whether it contains a bug. For example, a code snippet can be a call expression with two arguments, which enables a bug detector to decide whether the arguments are passed in the correct order.

There are various ways to implement a training data generator. For example, suppose the bugs of interest are accidentally swapped arguments of function calls. A training data generator for this bug pattern gathers positive examples by extracting all function calls that have at least two arguments and negative examples by permuting the order of these arguments of the function calls Under the assumption that the given code is mostly correct, the original calls are likely correct, whereas changing the order of arguments is likely to provide an incorrect call.

Our idea of artificially creating likely incorrect code relates to mutation testing [Jia and Harman 2011] and to work on artificially introducing security vulnerabilities [Dolan-Gavitt et al. 2016; Pewny and Holz 2016]. These existing techniques are intended for evaluating the effectiveness of test suites and of vulnerability detection tools, respectively. Our work differs by creating likely incorrect code for a different purpose, training a machine learning model, and by considering bug patterns that are amenable to name-based bug detection.

\subsection{Embeddings for Identifiers and Literals}

As machine learning relies on vector representations of the analyzed data, to learn a bug detector, we require vector representations of code snippets. An important challenge for a name-based bug detector, is to reason about identifier names, which are natural language information and therefore inherently difficult to understand for a computer. Our goal is to distinguish semantically similar identifiers from dissimilar ones. For example, the bug detector that searches for swapped arguments may learn from examples such as done(error, result) that done(res, err) is likely to be wrong, because error $\approx$ err and result $\approx$ res, where $\approx$ refers to semantic similarity. In contrast, seq and sequoia are semantically dissimilar because they refer to different concepts, even though they share a common prefix of characters. As illustrated by these examples, semantic similarity does not always correspond to lexical similarity, as considered by prior work [Liu et al. 2016; Pradel and Gross 2011; Rice et al. 2017], and may even exist cross type boundaries. To enable a machine learning-based bug detector to reason about identifiers and their semantic similarities, we require a representation of identifiers that preserves these semantic similarities.

In addition to identifiers, we also consider literals in code, such as true and 23, because they also convey relevant semantic information that can help to detect bugs. For example, true and 1 are similar (in JavaScript, at least) because both evaluate to true when being used in a conditional. To simplify the presentation, we say "identifier" to denote both identifiers and literals. Our implementation disambiguates tokens that represent identifiers and literals from each other and from language keywords by prepending the former with "ID:" and the latter with "LIT:".

DeepBugs reasons about identifiers by automatically learning a vector representation, called embeddings, for each identifier based on a corpus of code:

Definition 2.2 (Embeddings). The embeddings are a map $E: I \rightarrow \mathbb{R}^{e}$ that assigns to each identifier in the set $I$ of identifiers a real-valued vector in an $e$-dimensional space. 
A naïve representation is a local, or one-hot, encoding, where $e=|I|$ and where each vector returned by $E$ contains only zeros except for a single element that is set to one and that represents the specific identifier. Such a local representation fails to provide two important properties. First, to enable efficient learning, we require an embedding that stores many identifiers in relatively short vectors. Second, to enable DeepBugs to generalize across non-identical but semantically similar identifiers, we require an embedding that assigns a similar vector to semantically similar identifiers

Instead of a local embedding, we use a distributed embedding, where the information about an identifier is distributed across all elements of the vector returned by $E$. Our distributed embedding is inspired by word embeddings for natural languages, specifically by Word2Vec [Mikolov et al 2013a]. The basic idea of Word2Vec is that the meaning of a word can be derived from the various contexts in which this word is used. In natural languages, the context of an occurrence of a word in a sequence of words is the window of words preceding and succeeding the word.

We adapt this idea to source code by viewing code as a sequence of tokens and by defining the context of the occurrence of an identifier as its immediately preceding and succeeding tokens. Given a sequence of tokens $t_{1}, \ldots, t_{i}, \ldots, t_{k}$, where $t_{i}$ is an identifier, the approach considers a window of $w$ tokens around $t_{i}$, containing the $\frac{w}{2}$ tokens before $t_{i}$ and the $\frac{w}{2}$ tokens after $t_{i}$. As a default, we choose $w=20$ in our experiments.

We use the CBOW variant of Word2Vec [Mikolov et al. 2013a], which trains a neural network that predicts a token from its surrounding tokens. To achieve this goal, the network feeds the given information through a hidden layer of size $e$, from which the token is predicted. We use $e=200$ for our experiments. Once trained, the network has learned a semantics-preserving representation of each identifier, and we use this representation as the embedding $E$ of the identifier.

For efficiency during training, we limit the vocabulary $V$ of tokens, including identifiers and literals, to $|V|=10,000$ by discarding the least frequent tokens. To represent tokens beyond $V$, we use a placeholder "unknown". Section 5.7 shows that this way of bounding the vocabulary size covers the vast majority of all occurrences of identifiers and literals.

\subsection{Vector Representations of Positive and Negative Code Examples}

Given code snippets extracted from a corpus, our approach uses the embeddings for identifiers to represent each snippet as a vector suitable for learning:

Definition 2.3 (Code representation). Given a code snippet $c \in C$, its code representation $v \in \mathbb{R}^{n}$ is an $n$-dimensional real-valued vector that contains the embeddings of all identifiers in $c$.

Each bug detector built on top of the DeepBugs framework chooses a code representation suitable for the specific kind of code snippet (explained in detail in Section 3). For example, to detect bugs related to function arguments, the code representation may contain the embeddings of the function name and the arguments.

All bug detectors share the same technique for extracting names of expressions. Given an AST node $n$ that represents an expression, we extract name $(n)$ as follows:

- If $n$ is an identifier, return its name.

- If $n$ is a literal, return a string representation of its value.

- If $n$ is a this expression, return "this".

- If $n$ is an update expression that increments or decrements $x$, return name $(x)$.

- If $n$ is a member expression base.prop that accesses a property, return name(prop).

- If $n$ is a member expression base [ $k]$ that accesses an array element, return name(base).

- If $n$ is a call expression base.callee(..), return name(callee).

- For any other AST node $n$, do not extract its name. 
Table 2. Examples of identifier names and literals extracted for name-based bug detectors.

\begin{aligned} & \hline Expression Extracted name \\ & \hline list ID:list \\ & 23 LIT:23 \\ & this LIT:this \\ & i++ ID:i \\ & myObject.prop ID:prop \\ & myArray[5] ID:myArray \\ & nextElement() ID:nextElement \\ & db.allNames()[3] ID:allNames \\ & \hline\end{aligned}

Table 2 gives examples of names extracted from JavaScript expressions. We use the prefixes "ID:" and "LIT:" to distinguish identifiers and literals. The extraction technique is similar to that used in manually created name-based bug detectors [Liu et al. 2016; Pradel and Gross 2011; Rice et al. 2017], but omits heuristics to make the extracted name suitable for a lexical comparison of names. For example, existing techniques remove common prefixes, such as get to increase the lexical similarity between, e.g., getNames and names. Instead, DeepBugs identifies semantic similarities of names through learned embeddings.

\subsection{Training and Querying a Bug Detector}

Based on the vector representation of code snippets, a bug detector is a model that distinguishes between vectors that correspond to correct and incorrect code examples, respectively.

Definition 2.4 (Bug detector). A bug detector $D$ is a binary classifier $D: C \rightarrow[0,1]$ that predicts the probability that a code snippet $c \in C$ is an instance of a particular bug pattern.

Training a bug detector consists of two steps. At first, DeepBugs computes for each positive example $c_{\text {pos }} \in C_{\text {pos }}$ its vector representation $v_{\text {pos }} \in \mathbb{R}^{n}$, which yields a set $V_{\text {pos }}$ of vectors. Likewise, the approach computes the set $V_{n e g}$ from the negative examples $c_{n e g} \in C_{n e g}$. Then, we train the bug detector $D$ in a supervised manner by providing two kinds of input-output pairs: $\left(v_{\text {pos }}, 0\right)$ and $\left(v_{n e g}, 1\right)$. The output of the learned model can be interpreted as the probability that the given code snippet is incorrect. That is, the model is trained to predict that positive code examples are correct and that negative code examples are incorrect.

In principle, a bug detector can be implemented by any classification technique. We use a feedforward neural network with an input layer of a size that depends on the code representation provided by the specific bug detector, a single hidden layer of size 200, and an output layer with a single element that represents the probability computed by $D$. We apply a dropout of 0.2 to the input layer and the hidden layer. As the loss function, we use binary cross-entropy and train the network with the RMSprop optimizer for 10 epochs with batch size 100.

Given a sufficiently large set of training data, the bug detector will generalize beyond the training examples and one can query it with previously unseen code. To this end, DeepBugs extracts code snippets $C_{\text {new }}$ in the same way as extracting the positive training data. For example, for a bug detector that identifies swapped function arguments, the approach extracts all function calls including their unmodified arguments. Next, DeepBugs computes the vector representation of each example $c_{n e w} \in C_{n e w}$, which yields a set $V_{\text {new }}$. Finally, we query the trained bug detector $D$ with every $v_{\text {new }} \in V_{\text {new }}$ and obtain for each code snippet a prediction of the probability that it is incorrect. To report warnings about bugs to a developer, DeepBugs ranks all warnings by 
the predicted probability in descending order. In addition, one can control the overall number of warnings by omitting all warnings with a probability below a configurable threshold.

It is important to note that bug detectors built with DeepBugs do not require any heuristics or manually designed filters of warnings, as commonly used in existing name-based bug detectors [Liu et al. 2016; Pradel and Gross 2011; Rice et al. 2017]. For example, the start-of-the-art bug detector to detect accidentally swapped function arguments relies on a hard-coded list of function names for which swapping the arguments is expected, such as flip, transpose, or reverse [Rice et al. 2017] Instead of hard-coding such heuristics, which is time-consuming and likely incomplete, learned name-based bug detectors infer these kinds of exceptions from the training data.

\section{NAME-BASED BUG DETECTORS}

This section presents three examples of name-based bug detectors built on top of the DeepBugs framework. The bug detectors address a diverse set of programming mistakes: accidentally swapped function arguments, incorrect binary operators, and incorrect operands in binary expressions. While the first bug pattern has been the target of previous work for statically typed languages [Liu et al. 2016; Pradel and Gross 2011; Rice et al. 2017], we are not aware of a name-based bug detector for the other two bug patterns. Implementing new bug detectors is straightforward, and we envision future work to create more instances of our framework, e.g., based on bug patterns mined from version histories [Brown et al. 2017; Hanam et al. 2016].

Each bug detector consists of two simple ingredients.

- Training data generator. A training data generator that traverses the code corpus and extracts positive and negative code examples for the particular bug pattern based on a code transformation (Definition 2.1). We find a simple AST-based traversal and transformation to be sufficient for all studied bug patterns.

- Code representation. A mapping of each code example into a vector that the machine learning model learns to classify as either benign or buggy (Definition 2.3). All bug detectors presented here build on the same embeddings of identifier names, allowing us to amortize the one-time effort of learning an embedding across different bug detectors.

Given these two ingredients and a corpus of training code, our framework learns a bug detector that identifies programming mistakes in previously unseen code.

The remainder of this section presents three bug detectors build on top of DeepBugs.

\subsection{Swapped Function Arguments}

The first bug detector addresses accidentally swapped arguments. This kind of mistake can occur both in statically typed and dynamically typed languages. For statically typed languages, this kind of bug occurs for methods that accept multiple equally typed arguments. For dynamically typed languages, the problem is potentially more widespread because all calls that pass two or more arguments are susceptible to the mistake due to the lack of static type checking. Example 1 in Table 1 shows a real-world example of this bug pattern.

Training Data Generator. To create training examples from given code, the approach traverses the AST of each file in the code corpus and visits each call site that has two or more arguments. For each such call site, the approach extracts the following information:

- The name $n_{\text {callee }}$ of the called function.

- The names $n_{a r g 1}$ and $n_{a r g 2}$ of the first and second argument.

- The name $n_{\text {base }}$ of the base object if the call is a method call, or an empty string otherwise.

- For arguments that are literals, the types $t_{a r g 1}$ and $t_{a r g 2}$ of the first and second argument, or empty strings if the arguments are values other than literals. 
- The names $n_{\text {param } 1}$ and $n_{\text {param } 2}$ of the formal parameters of the called function, or empty strings if unavailable.

All names are extracted using the name function defined in Section 2.4. Type information is used only for literals because JavaScript is a dynamically typed language. The extracted types correspond to the built-in types of JavaScript (more specifically, ECMAScript 5 [ECMA 2011]): "string", e.g., for a literal "abc", "number", e.g., for a literal 23, "boolean" for the literals true and false, "RegExp" for regular expression literals, and "null" for null. An addition, we consider undefined values to have type "undefined". This information is available only for a small subset of all code locations analyzed by DeepBugs. For example, among all calls analyzed for swapped arguments, only $18 \%$ have a known type for one and only $4 \%$ have a known type for both arguments. To obtain the names of the formal parameters of the called function, we resolve function calls heuristically, as sound static call resolution is non-trivial in JavaScript. If either $n_{\text {callee }}, n_{\text {arg } 1}$, or $n_{\text {arg } 2}$ are unavailable, e.g., because the name function cannot extract the name of a complex expression, then the approach ignores this call site.

From the extracted information, the training data generator creates for each call site a positive example

$$
x_{\text {pos }}=\left(n_{\text {base }}, n_{\text {callee }}, n_{\text {arg } 1}, n_{\text {arg } 2}, t_{\text {arg } 1}, t_{\text {arg } 2}, n_{\text {param } 1}, n_{\text {param } 2}\right)
$$

and a negative example

$$
x_{n e g}=\left(n_{\text {base }}, n_{\text {callee }}, n_{\text {arg } 2}, n_{\text {arg1 } 1}, t_{\text {arg } 2}, t_{\text {arg } 1}, n_{\text {param } 1}, n_{\text {param } 2}\right) .
$$

That is, to create the negative example, we simply swap the arguments w.r.t. the order in the original code.

Code representation. To enable DeepBugs to learn from the positive and negative examples, we transform $x_{p o s}$ and $x_{n e g}$ from tuples of strings into vectors. To this end, the approach represents each string in the tuple $x_{p o s}$ or $x_{n e g}$ as a vector. Each name $n$ is represented as $E(n)$, where $E$ is the learned embedding from Section 2.3. To represent type names as vectors, we define a function $T$ that maps each built-in type in JavaScript to a randomly chosen binary vector of length 5 . For example, the type "string" may be represented by a vector $T$ (string) $=[0,1,1,0,0]$, whereas the type "number" may be represented by a vector $T$ (number) $=[1,0,1,1,0]$. Finally, based on the vector representation of each element in the tuple $x_{p o s}$ or $x_{n e g}$, we compute the code representation for $x_{p o s}$ or $x_{n e g}$ as the concatenation the individual vectors.

\subsection{Wrong Binary Operator}

The next two bug detectors address mistakes related to binary operations. At first, we consider code that accidentally uses the wrong binary operator, e.g., $i<=$ length instead of $i<$ length Such mistakes are hard to find, especially in a dynamically typed language, but identifier names can provide valuable hints when search these mistakes.

Training Data Generator. The training data generator traverses the AST of each file in the code corpus and extracts the following information from each binary operation:

- The names $n_{\text {left }}$ and $n_{\text {right }}$ of the left and right operand.

- The operator op of the binary operation.

- The types $t_{\text {left }}$ and $t_{\text {right }}$ of the left and right operand if they are literals, or empty strings otherwise.

- The kind of AST node $k_{\text {parent }}$ and $k_{\text {grandP }}$ of the parent and grand-parent nodes of the AST node that represents the binary operation. 
We extract the (grand-)parent nodes to provide some context about the binary operation to DeepBugs, e.g., whether the operation is part of a conditional or an assignment. If either $n_{\text {left }}$ or $n_{\text {right }}$ are unavailable, then we ignore the binary operation.

From the extracted information, the approach creates a positive and a negative example:

$$
\begin{aligned}
& x_{\text {pos }}=\left(n_{\text {left }}, n_{\text {right }}, o p, t_{\text {left }}, t_{\text {right }}, k_{\text {parent }}, k_{\text {grand } P}\right) \\
& x_{\text {neg }}=\left(n_{\text {left }}, n_{\text {right }}, o p^{\prime}, t_{\text {left }}, t_{\text {right }}, k_{\text {parent }}, k_{\text {grand } P}\right)
\end{aligned}
$$

The operator $o p^{\prime} \neq o p$ is a randomly selected binary operator different from the original operator. For example, given a binary expression $i<=$ length, the approach may create a negative example $i<$ length or $i$ length, which is likely to create incorrect code.

Code representation. Similar to the above bug detector, we create a vector representation of each positive and negative example by mapping each string in the tuple to a vector and by concatenating the resulting vectors. To map a kind of AST node $k$ to a vector, we use a map $K$ that assigns to each kind of AST node in JavaScript a randomly chosen binary vector of length 8 .

\subsection{Wrong Operand in Binary Operation}

The final bug detector addresses code that accidentally uses an incorrect operand in a binary operation. The intuition is that identifier names help to decide whether an operand fits another given operand and a given binary operator. For example, the bug detector may identify the $x$ operand in height - $\mathrm{x}$ as possibly buggy because the operation was intended to be height - $\mathrm{y}$.

Training Data Generator. The training data generator extracts the same information as in Section 3.2, and then replaces one of the operands with a randomly selected alternative. That is, the positive example is

$$
x_{\text {pos }}=\left(n_{\text {left }}, n_{\text {right }}, \text { op, } t_{\text {left }}, t_{\text {right }}, k_{\text {parent }}, k_{\text {grandP }}\right)
$$

whereas the negative example is either

$$
x_{\text {neg }}=\left(n_{\text {left }}^{\prime}, n_{\text {right }}, \text { op }, t_{\text {left }}^{\prime}, t_{\text {right }}, k_{\text {parent }}, k_{\text {grandP }}\right)
$$

or

$$
x_{\text {neg }}=\left(n_{\text {left }}, n_{\text {right }}^{\prime}, \text { op }, t_{\text {left }}, t_{\text {right }}^{\prime}, k_{\text {parent }}, k_{\text {grandP }}\right) .
$$

The name and type $n_{\text {left }}^{\prime}$ and $t_{\text {left }}^{\prime}$ (or $n_{\text {right }}^{\prime}$ and $t_{\text {right }}^{\prime}$ ) are different from those in the positive example. To create negative examples that a programmer might also create by accident, we use alternative operands that occur in the same file as the binary operation. For example, given bits $<<2$, the approach may transform it into a negative example bits $\ll$ next, which is likely to yield incorrect code.

Code representation. The vector representation of the positive and negative examples is the same as in Section 3.2.

\section{IMPLEMENTATION}

The code extraction and generation of training examples is implemented as simple AST traversals based on the Acorn JavaScript parser. ${ }^{2}$ The training data generator writes all extracted data into text files. These files are then read by the implementation of the bug detector, which builds upon the TensorFlow and Keras frameworks for deep learning. ${ }^{3}$ The large majority of our implementation is

\footnotetext{
${ }^{2}$ https://github.com/ternjs/acorn

${ }^{3}$ https://www.tensorflow.org/ and https://keras.io/
} 
Table 3. Statistics on extraction and generation of training data.

\begin{tabular}{lrr}
\hline \multirow{2}{*}{ Bug detector } & \multicolumn{2}{c}{ Examples } \\
\cline { 2 - 3 } & Training & Validation \\
\hline Swapped arguments & $1,450,932$ & 739,188 \\
Wrong binary operator & $4,901,356$ & $2,322,190$ \\
Wrong binary operand & $4,899,206$ & $2,321,586$ \\
\hline
\end{tabular}

in the generic framework, whereas the individual bug detectors are implemented in about 100 lines of code each.

\section{EVALUATION}

We evaluate DeepBugs by applying it to a large corpus of JavaScript code. Our main research questions are:

- How effective is the approach at distinguishing correct from incorrect code?

- Does the approach find bugs in production JavaScript code?

- How long does it take to train a model and, once a model has been trained, to predict bugs?

- How useful are the learned embeddings of identifiers compared to a simpler vector representation?

\subsection{Experimental Setup}

As a corpus of code, we use 150,000 JavaScript files provided by the authors of earlier work [Raychev et al. 2016b]. The corpus contains files collected from various open-source projects and has been cleaned by removing duplicate files. In total, the corpus contains 68.6 million lines of code. We use 100,000 files for training and the remaining 50,000 files for validation. For learning the embeddings and for determining the top 10,000 identifiers that are part of our vocabulary, we use only the training files. The split of the corpus into training and validation data is the same as in earlier work [Raychev et al. 2016b]. It ensures that the two sets of files are disjoint, but not that the projects that the files come from are disjoint. All experiments are performed on a single machine with 48 Intel Xeon E5-2650 CPU cores, 64GB of memory, and a single NVIDIA Tesla P100 GPU.

\subsection{Extraction and Generation of Training Data}

Table 3 summarizes the training and validation data that DeepBugs extracts and generates for the three bug detectors. Each bug detector learns from several millions of examples, which is sufficient for effective learning. Half of the examples are positive and negative code examples, respectively. Manually creating this amount of training data, including negative examples, would be impractical, showing the benefit of our automated data generation approach.

\subsection{Warnings in Real-World Code}

To evaluate the effectiveness of bug detectors built with DeepBugs, we conduct two sets of experiments. First, reported in the following, we apply the bug detectors to unmodified real-world code and manually inspect the reported warnings to assess the precision of the learned bug detectors. Second, reported in Section 5.4, we conduct a large-scale evaluation with hundreds of thousands of artificially created bugs, which allows us to study the accuracy, recall, and precision of each learned bug detector. 
Table 4. Results of inspecting and classifying warnings in real-world code.

\begin{tabular}{lrrrr}
\hline Bug detector & \multicolumn{3}{c}{ Reported Bugs Code quality } & $\begin{array}{r}\text { False } \\
\text { problem positives }\end{array}$ \\
\hline Swapped arguments & 50 & 23 & 0 & 27 \\
Wrong binary operator & 50 & 37 & 7 & 6 \\
Wrong binary operand & 50 & 35 & 0 & 15 \\
\hline Total & 150 & 95 & 7 & 48 \\
\hline
\end{tabular}

To study the effectiveness of DeepBugs on real-world code, we train each bug detector with the 100,000 training files, then apply the trained bug detector to the 50,000 validation files, and finally inspect code locations that the bug detectors report as potentially incorrect. For the manual inspection, we sort all reported warnings by the probability that the code as incorrect, as reported by the classifier, and then inspect the top 50 warnings per bug detector. Based on the inspection, we classify each warning in one of three categories:

- Bug. A warning points to a bug if the code is incorrect in the sense that it does not result in the expected runtime behavior.

- Code quality problem. A warning points to a code quality problem if the code yields the expected runtime behavior but should nevertheless be changed to be less error-prone or be more efficient. This category includes code that violates widely accepted conventions and programming rules traditionally checked by static linting tools. Our learned bug detectors find code quality problems because such problems often correspond to unusual code that diverges from a common coding idiom.

- False positive. A warning is a false positive in all other cases. If we are unsure about the intended behavior of a particular code location, we conservatively count it as a false positive. We also encountered various code examples with misleading identifier names, which we classify as false positives because the decision whether an identifier is misleading is rather subjective.

Table 4 summarizes the results of inspecting and classifying warnings. Out of the 150 inspected warnings, 95 warnings point to bugs and 7 warnings point to a code quality problem, i.e., $68 \%$ of all warnings point to an actual problem. Existing manually created bug detectors typically provide similar true positives rates, but heavily rely on heuristics to filter likely false positives. We conclude that our learned name-based bug detectors are effective at finding real-world bugs while providing high precision.

5.3.1 Examples of Bugs. Many of the detected problems are difficult to detect with a traditional, name-unaware analysis, because the programming mistake is obvious only when understanding the intended semantics of the involved identifiers and literals. The three motivating examples in Table 1 are bugs detected by DeepBugs. We discuss three more representative examples in the following.

Buggy call of Promise. done. The following code is from Apigee's JavaScript SDK. The Promise. done function expects an error followed by a result, but the second call of done passes the arguments the other way around.

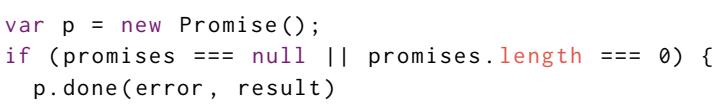




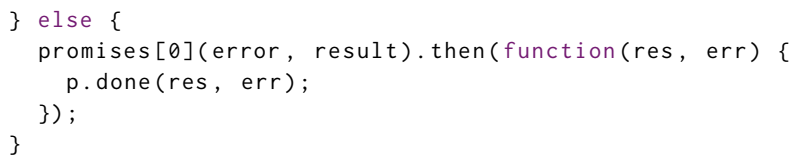

Meaningless binary operation. The following code from RequirefS is a bug that was found and fixed independently of us. ${ }^{4}$ The binary operation ' ${ }^{\prime}===0$ is obviously meaningless, as it always returns false. Instead, the developer intended to write indexOf('-') $===0$. DeepBugs detects this problem because it handles literals the same way as identifiers.

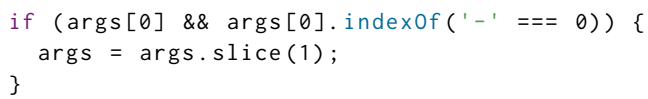

Buggy call of assertEquals. The following code is from the test suite of the Google Closure library. The arguments of assertEquals are supposed to be the expected and the actual value of a test outcome. Swapping the argument leads to an incorrect error message when the test fails, which makes debugging unnecessarily hard. Google developers consider this kind of mistake a bug [Rice et al. 2017].

assertEquals(tree.remove('merry'), null);

\subsubsection{Examples of Code Quality Problems.}

Error-prone termination condition. The following code from Phaser.js is correct but using !==, instead of $<$, as the termination condition of a for-loop is generally discouraged. The reason is that the loop risks to run out-of-bounds when the counter is incremented by more than one or assigned an out-of-bounds value, e.g., by an accidental assignment in the loop body. DeepBugs finds this problem because most code follows the convention of using $<$ in the termination condition.

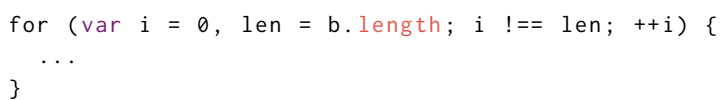

Error-prone and inefficient logical OR. The following code from the promiscuous library uses the bitwise OR operator I to compare two boolean values. Unfortunately, using bitwise instead of logical operators for booleans is known to be inefficient ${ }^{5}$ and may cause unexpected behavior for non-boolean operands that are supposed to be coerced into booleans. DeepBugs reports this problem because the is identifiers suggest two boolean return values, which are usually compared through a logical operation, such as || .

transform = value \&\& (is (obj, value) $\mid$ is (func, value)) \&\& value. then;

5.3.3 Examples of False Positives. We discuss some representative examples of false positives. Some of them are due to wrapper functions, e.g., Math.max, for which our approach extracts a generic name, "max", that does not convey the specific meaning of the value returned by the call expression. The main reason for false positives, though, are poorly named variable names that lead to surprisingly looking but correct code. For example, DeepBugs falsely reports a warning claiming that the each operand in the following statement is wrong:

var $c w=c s[i] . w i d t h+$ each;

\footnotetext{
$\overline{{ }^{4} \text { https://github.com/requirejs/requirejs/issues/700 }}$

${ }^{5}$ https://jsperf.com/bitwise-logical-and
} 
A close inspection of the code reveals that the operand is correct, despite the fact that the name each may not be an ideal choice for a number added to a width. Another example is the following comparison of value. length and is:

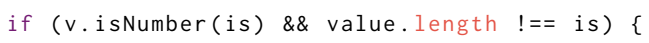

Our "wrong binary operand" bug detector reports a warning about the is operand, because this name is typically used for booleans and comparing a boolean to a value stored in length is very unusual. Again, close inspection of the code shows it to be correct, though. These false positives illustrate an inherent limitation of name-based bug detection: Because the approach relies in reasonable names that are in line with common practice, it may fail when developers choose to diverge from the norm. Overall, we find these cases to be rare enough to yield a bug detector with a precision of $68 \%$.

5.3.4 Comparison with Traditional Static Checkers. There are various existing static bug detection tools, e.g., Google's Error Prone [Aftandilian et al. 2012], Facebook's Infer [Calcagno et al. 2015] or FindBugs [Hovemeyer and Pugh 2004]. Since DeepBugs targets code written in JavaScript, we compare it with JSHint ${ }^{6}$, a popular, lint-like static checker for JavaScript. We apply JSHint on the files containing the 102 true positives detected by DeepBugs (Table 4). For each warning reported by JSHint on a problematic line, we manually check whether the warning points to the problem detected by DeepBugs (as opposed to, e.g., another, unrelated warning that coincidentally is at the same line).

JSHint finds seven out of the 102 problems. All seven problems detected by JSHint originate from a single kind of warning: a non-callable value that is found on the right-hand side of instanceof, e.g. someExpression instanceof null, which always yields false. We conclude that DeepBugs and JSHint are mostly complementary.

\subsection{Accuracy and Recall of Bug Detectors}

In the following, we evaluate the accuracy and recall of each bug detector at a large scale based on automatically seeded bugs. This part of our evaluation complements the manual inspection of potential real-world bugs (Section 5.3) with an automated assessment based on a large number of artificially seeded bugs. Informally, accuracy here means how many of all the classification decisions that the bug detector makes are correct. Recall means how many of all bugs in a corpus of code that the bug detector finds. To evaluate these metrics, we train each bug detector on the 100,000 training files and then apply it to the 50,000 validation files. For the validation files, we use the training data generator to extract correct code examples $C_{p o s}$ and to artificially create likely incorrect code examples $C_{n e g}$. We then query the bug detector $D$ with each example $c$, which yields a probability $D(c)$ that the example is buggy. Finally, we compute the accuracy is as follows:

$$
\text { accuracy }=\frac{\left|\left\{c \mid c \in C_{\text {pos }} \wedge D(c)<0.5\right\}\right|+\left|\left\{c \mid c \in C_{n e g} \wedge D(c) \geq 0.5\right\}\right|}{\left|C_{\text {pos }}\right|+\left|C_{n e g}\right|}
$$

The last column of Table 5 shows the accuracy of the bug detectors. The accuracy ranges between $89.06 \%$ and $94.70 \%$, i.e., all bug detectors are highly effective at distinguishing correct from incorrect code examples.

The recall of a bug detector is influenced by how many warnings the detector reports. More warnings are likely to reveal more bugs, thus increasing recall, but are also more likely to report false positives. Moreover, in practice, developers are only willing to inspect some number of warnings.

$\overline{{ }^{6} \text { http://jshint.com/ }}$ 
Table 5. Accuracy of the bug detectors with random and learned embeddings for identifiers.

\begin{tabular}{lrr}
\hline & \multicolumn{2}{c}{ Embedding } \\
\cline { 2 - 3 } & Random & Learned \\
\hline Swapped arguments & $93.88 \%$ & $94.70 \%$ \\
Wrong binary operator & $89.15 \%$ & $92.21 \%$ \\
Wrong binary operand & $84.79 \%$ & $89.06 \%$ \\
\hline
\end{tabular}

To measure recall, we assume that a developer inspects all warnings where the probability $D(c)$ is above some threshold. We model this process by turning the bug detector $D$ into a boolean function:

$$
D_{t}(c)=\left\{\begin{array}{lll}
1 & \text { if } \quad D(c)>t \\
0 & \text { if } \quad D(c) \leq t
\end{array}\right.
$$

where $t$ is a configurable threshold that controls how many warnings to report. Based on $D_{t}$, we compute recall as follows:

$$
\text { recall }=\frac{\left|\left\{c \mid c \in C_{n e g} \wedge D_{t}(c)=1\right\}\right|}{\left|C_{n e g}\right|}
$$

Furthermore, we measure the number of false positives as follows:

$$
\# f p s=\left|\left\{c \mid c \in C_{p o s} \wedge D_{t}(c)=1\right\}\right|
$$

Note that both recall and the number of false positives are estimates based exclusively on artificially seeded bugs. The recall measure assumes that all seeded bugs are actual bugs that should be detected. The false positive measure assumes that all of the original code is correct and that each warning in $C_{\text {pos }}$ should therefore be counted as a false positive. We consider these measures here because they allow us to evaluate DeepBugs with hundreds of thousands of artificial bugs, which complements the evaluation with real-world bugs in Section 5.3.

Figure 2 shows the recall of the three bug detectors as a function of the threshold for reporting warnings. Each plot shows the results for five different thresholds: $t \in\{0.5, \ldots, 0.9\}$. As expected, the recall decreases when the threshold increases, because fewer warnings are reported and therefore some bugs are missed. The results also show that some bug detectors are more likely than others to detect a bug, if a bug is present.

Considering more warnings naturally also leads to more false positives. For a threshold of $t=0.5$, the three bug detectors report a total of 116,941 warnings, which corresponds to roughly one warning per 196 lines of code. In contrast, for a threshold of $t=0.9$, the number reduces to 11,292, i.e., roughly one warning per 2,025 lines of code. In practice, we expect developers to inspect only the top-ranked warnings, as we do in Section 5.3.

\subsection{Efficiency}

Table 6 shows how long it takes to train a bug detector and to use it to predict bugs in previously unseen code. The training time consists of the time to gather code examples and of time to train the classifier. The prediction time also consist of the time to extract code examples and of the time to query the classifier with each example. Running both training and prediction on all 150,000 files takes between 36 minutes and 73 minutes per bug detector. The average prediction time per JavaScript file is below 20 milliseconds. We conclude that the training time is reasonable for a machine learning approach and that, once a bug detector is trained, using it on new code takes very little time. 

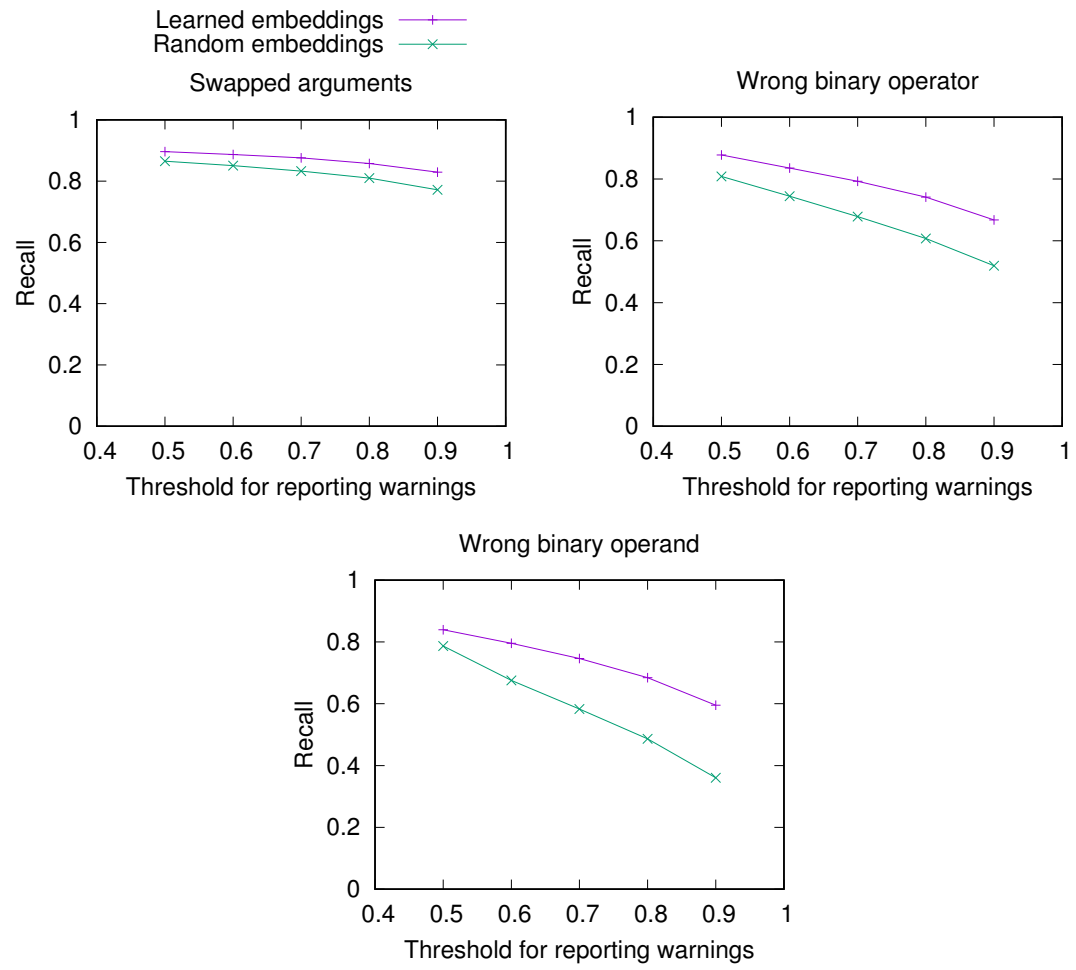

Fig. 2. Recall of the bug detectors with different thresholds $t$ for reporting warnings. Each plot contains data points obtained with $t \in\{0.5, \ldots, 0.9\}$. The data labeled "Learned embedding" corresponds to the DeepBugs approach.

Table 6. Time ( $\min : \mathrm{sec}$ ) required for training and using a bug detector across the entire code corpus.

\begin{tabular}{lrr|rr}
\hline Bug detector & \multicolumn{2}{c}{ Training } & \multicolumn{2}{c}{ Prediction } \\
\cline { 2 - 5 } & Extract & Learn & Extract & Predict \\
\hline Swapped arguments & $7: 46$ & $20: 48$ & $2: 56$ & $5: 10$ \\
Wrong bin. operator & $2: 44$ & $49: 47$ & $1: 28$ & $8: 39$ \\
Wrong bin. operand & $2: 44$ & $56: 35$ & $1: 28$ & $12: 14$ \\
\hline
\end{tabular}

\subsection{Usefulness of Embeddings}

Our work distinguishes itself from previous name-based bug detectors by reasoning about identifiers based on a semantic representation, i.e., the learned embeddings (Section 2.3). We evaluate the usefulness of learned embeddings quantitatively, qualitatively, and based on their effect on the bugs detected by DeepBugs. To this end, we compare DeepBugs with learned embeddings to a simpler variant of the approach that uses a baseline vector representation. The baseline assigns to each identifier considered by DeepBugs a unique, randomly chosen binary vector of length $e$, i.e., the same length as our learned embeddings. 


\begin{aligned} & \hline \multicolumn{2}{c}{ ID:name } \\ & \hline Simil. Identifier \\ & \hline 0.4 ID:names \\ & 0.4 ID:getName \\ & 0.39 ID:_name \\ & 0.39 LIT:Identifier \\ & 0.37 ID:fullName \\ & 0.36 ID:property \\ & 0.35 ID:type \\ & 0.34 LIT:: \\ & 0.34 ID:Identifier \\ & 0.34 ID:namespace \\ & \hline\end{aligned}

\begin{aligned} & \hline \multicolumn{2}{c}{ ID:wrapper } \\ & \hline Simil. Identifier \\ & \hline 0.36 ID:wrap \\ & 0.36 ID:_wrapped \\ & 0.36 ID:wrapInner \\ & 0.34 ID:element \\ & 0.32 ID:container \\ & 0.32 ID:wrapAll \\ & 0.31 ID:attribs \\ & 0.31 ID:\$wrapper \\ & 0.3 LIT:rect \\ & 0.3 ID:renderer \\ & \hline\end{aligned}

\begin{aligned} & \hline \multicolumn{2}{c}{ ID:msg } \\ & \hline Simil. Identifier \\ & \hline 0.57 ID:message \\ & 0.46 ID:error \\ & 0.4 LIT:error \\ & 0.39 ID:receive \\ & 0.39 LIT:msg \\ & 0.39 LIT:Error \\ & 0.36 ID:alert \\ & 0.36 LIT:: \\ & 0.35 LIT:message \\ & 0.34 LIT:log \\ & \hline\end{aligned}

Fig. 3. Most similar identifiers according to the learned embeddings.

5.6.1 Quantitative Evaluation. We compare the learned embeddings with the baseline w.r.t. accuracy and recall. Table 5 shows in the "Random" column what accuracy the bug detectors achieve with the baseline. Compared to the accuracy with the random embeddings, the learned embeddings yield a more accurate classifier.

Figure 2 compares the recall of the bug detectors with the two embeddings. We find that, for all three bug detectors, the learned embeddings increase the recall. The reason is that the learned embeddings enable a bug detector to reason about semantic similarities between syntactically different code examples, which enables it to learn and predict bugs across similar examples. We conclude from these results that the learned embeddings improve the effectiveness of DeepBugs. At the same time, the bug detectors achieve relatively high accuracy and recall even with randomly created embeddings, showing that the overall approach has value even when no learned embeddings are available.

5.6.2 Qualitative Evaluation. For a qualitative assessment of the embeddings, we show for a set of identifiers which other identifiers are the most similar according to the learned embeddings. Figure 3 shows the ten most similar identifiers for name, wrapper, and msg. Recall that the "ID:" and "LIT:" prefixes are for distinguishing identifiers from literals. The similarity scores range between 0 and 1 , where 1 means the maximum similarity.

The examples illustrate three observations. First, the embeddings encode similarities between abbreviated and non-abbreviated identifiers, e.g., msg and message. This property is useful because abbreviations are common in source code and often introduce words not found in natural language documents. Second, the embeddings encode similarities between lexically similar identifiers, e.g., name and getName. Previous work on name-based bug detection relies on traditional string similarity metrics, e.g,. Levenshtein distance, and on heuristics, such as removing the get prefix, to capture these similarities. Instead, we here learn the similarities from existing code. Finally and perhaps most importantly, the embeddings encode semantic similarities between lexically dissimilar identifiers, such as name and Identifier, or wrapper and container. Overall, we conclude that learned embeddings encode at least some semantic similarities of identifiers, which helps understand why a learning approach to name-based bug detection is effective.

5.6.3 Effect on Detected Real-World Bugs. We measure how the embeddings affect the bugs detected by DeepBugs by obtaining a list of warnings with a variant of the approach that uses the baseline, 


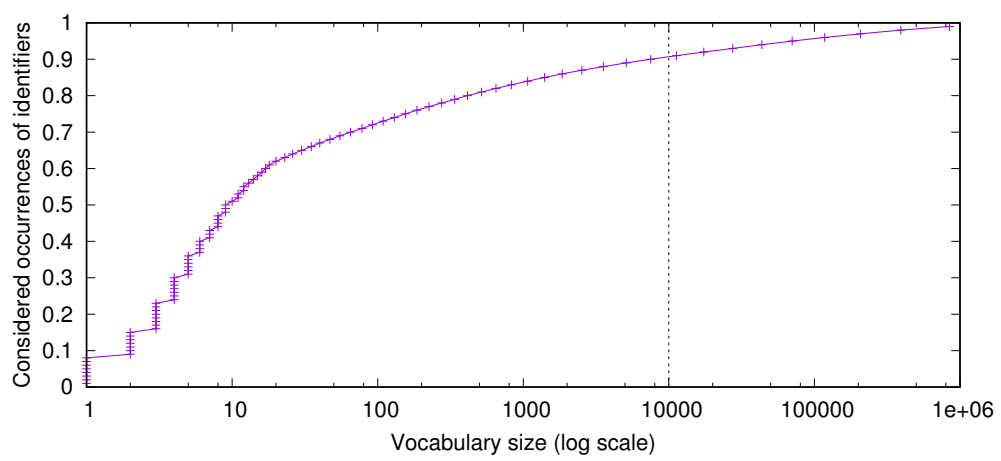

Fig. 4. Number of considered identifier occurrences depending on vocabulary size.

random vector representation and by comparing this list to the warnings in real-world code inspected in Section 5.3. To this end, we check for each true positive, i.e., bug or code quality problem, in Table 4 whether it is also found with random embeddings. We consider a true positive to be found with the baseline embeddings if the code location is predicted as buggy with a probability of at least 0.95 , assuming that developers would focus on such high-confidence warnings when using DeepBugs in practice. When using DeepBugs with learned embeddings, all inspected warnings in Table 4 have a predicted probability of at least 0.99 , i.e., considering warnings with a confidence of at least 0.95 for the baseline conservatively overapproximates the baseline's bug detection abilities.

We find that out of the 102 true positives detected by DeepBugs, 11 are missed when using the baseline embeddings instead of the learned embeddings. Specifically, the baseline approach misses 2 true positives related to swapped arguments, 6 true positives related to wrong binary operators, and 3 true positives related to wrong binary operands. For example, relying on the random embeddings causes the approach to miss two code quality problems similar to an example discussed in Section 5.3.2: The code is of the form is (..) | is (..), i.e., it uses a bitwise OR operator where the developer meant to express a logical OR, which is error-prone and inefficient, as discussed above. Providing learned embeddings to DeepBugs detects such problems because the classifier learns that bitwise OR is very uncommon for booleans and because the learned embeddings suggest that the identifier is usually represents a boolean. Only by putting these two pieces of information together, DeepBugs is able to report a warning. In contrast, when using random embeddings, the approach does not have a strong enough signal that is might represent a boolean value and therefore misses these warnings.

Overall, these results are consistent with the quantitative evaluation of the usefulness of embeddings, and in particular with the recall results in Figure 2: Learning embeddings is not essential for the approach, but it helps to detect bugs not found otherwise.

\subsection{Vocabulary Size}

An important parameter of our approach is the size $|V|$ of the vocabulary of tokens considered for learning embeddings (Section 2.3). Considering a larger vocabulary enables DeepBugs to reason about more code locations but also increases the resource requirements for learning the embeddings and risks to reduce accuracy due to very uncommon identifiers for which not enough training data is available. The total number of unique tokens in the training data set is about 2.4 million, almost all of which are either identifiers or literals. For our experiments, we set $|V|=10,000$, i.e., we consider the 10,000 most frequent tokens and replace all others with an "unknown" placeholder. 
The reason why such a relatively small vocabulary size is effective is illustrated in Figure 4. For a given vocabulary size, the figure shows the number of occurrences of identifiers that are included in the vocabulary. Note that the horizontal axis uses a logarithmic scale. The figure shows that a small number of tokens covers a large percentage of the occurrences of tokens. The reason is that some tokens are very frequent, while others occur extremely rarely. The dashed line is our default vocabulary size, which covers over $90 \%$ of all occurrences of tokens.

\section{RELATED WORK}

\subsection{Machine Learning and Language Models for Code}

The recent successes in machine learning have lead to a strong interest in applying machine learning techniques to programs. Existing approaches address a variety of development tasks, including code completion based on probabilistic models of code [Bielik et al. 2016; Raychev et al 2016a, 2014], predicting fixes of compilation errors via neural networks [Bhatia and Singh 2016; Gupta et al. 2017], the generation of inputs for fuzz testing via probabilistic, generative language models [Patra and Pradel 2016] or neural networks [Amodio et al. 2017; Godefroid et al. 2017; Liu et al. 2017], and inferring thread safety documentation using graph-based learning [Habib and Pradel 2018]. Deep neural networks also help recommend API usages [Gu et al. 2016], detect code clones [White et al. 2016], classify programs into pre-defined categories [Mou et al. 2016], and adapt copied-and-pasted code to its surrounding code [Allamanis and Brockschmidt 2017]. A recent survey discusses more approaches [Allamanis et al. 2017a]. All these approaches exploit the availability of a large number of examples to learn from, e.g., in the form of publicly available code repositories, and that source code has regularities even across projects written by different developers [Hindle et al. 2012]. We also exploit this observation but for a different task, bug finding, than the above work. Another contribution of our work is to augment the training data provided by the existing code by generating negative training examples through simple code transformations.

Name-based bug detection relates to existing learning-based approaches that consider identifiers. JSNice [Raychev et al. 2015] and JSNaughty [Vasilescu et al. 2017] address the problem of recovering meaningful identifiers from minified code, using conditional random fields and statistical machine translation, respectively. Another approach summarizes code into descriptive names that can serve, e.g., as method names [Allamanis et al. 2016]. The Naturalize tool suggests more natural identifiers based on an n-gram model [Allamanis et al. 2014]. Applying such a tool before running our name-based bug detectors is likely to improve its effectiveness.

A recent graph-based learning approach [Allamanis et al. 2017b] applies gated graph neural networks to an augmented AST representation of code, and shows that a learned model can predict how to name a variable and which variable to use in a given context. To learn representations of code, their approach relies on static type information, which is not available in our setting.

Word embeddings, e.g., Word2Vec approach [Mikolov et al. 2013b], are widely used in natural language processing, which has inspired our embeddings of identifiers. Other recent work has proposed embeddings for source code, e.g., for API methods [Nguyen et al. 2017], functions [DeFreez et al. 2018], for terms that occur both in programs and natural language documentation [Ye et al. 2016], as well as a generic, AST-based representation of code [Alon et al. 2018]. Our evaluation shows that a learned embedding is beneficial for the problem of bug detection. Incorporating another embedding of identifiers than our Word2Vec-based embedding into our framework is straightforward. 


\subsection{Machine Learning and Language Models for Analyzing Bugs}

Even though it has been noted that buggy code stands out compared to non-buggy code [Ray et al. 2016], little work exists on automatically detecting bugs via machine learning. Murali et al. train a recurrent neural network that probabilistically models sequences of API calls and then use it for finding incorrect API usages [Murali et al. 2017]. In contrast to DeepBugs, their model learns from positive examples only and focuses on bug patterns that can be expressed via probabilistic automata. Bugram detects bugs based on an n-gram model of code. Similar to the above, it learns only from positive examples.

Choi et al. [je Choi et al. 2017] train a memory neural network [Weston et al. 2014] to classify whether code may produce a buffer overrun. The VulDeePecker approach [Li et al. 2018] trains a neural network to determine whether a code snippet suffers from specific kinds of vulnerabilities. Their models learn from positive and negative examples, but the examples are manually created and labeled. A key insight of our work is that simple code transformations that seed artificial bugs provide many negative examples, and that learning from these examples yields a classifier that is effective for real-world code.

Finally, Wang et al. use a deep belief network to find a vector representation of ASTs, which are used for defect prediction [Wang et al. 2016]. Their approach marks entire files as likely to (not) contain a bug. However, in contrast to our and other bug finding work, their approach does not pinpoint the buggy location.

\subsection{Bug Finding}

A related line of research is specification mining [Ammons et al. 2002] and the use of mined specifications to detect unusual and possibly incorrect code [Liang et al. 2016; Pradel et al. 2012; Wasylkowski and Zeller 2009]. In contrast to our work, these approaches learn only from correct examples [Hangal and Lam 2002] and then flag any code that is unusual compared to the correct examples as possibly incorrect, or search for inconsistencies within a program [Engler et al. 2001]. Our work replaces the manual effort of creating and tuning such approaches by learning and does so effectively by learning from both correct and buggy code examples.

Our name-based bug detectors are motivated by manually created name-based program analyses [Liu et al. 2016; Pradel and Gross 2011; Rice et al. 2017]. The "swapped arguments" bug detector is inspired by the success of a manually developed and tuned name-based bug detector for this kind of bug [Rice et al. 2017]. For the other two bug detectors, we are not aware of any existing approach that addresses these problems based on identifiers. Our work differs from existing name-based bug detectors (i) by exploiting semantic similarities that may not be obvious to a lexical comparison of identifiers, (ii) by replacing manually tuned heuristics to improve precision and recall with automated learning from examples, and (iii) by applying name-based bug detection to a dynamically typed language.

There are various bug detection techniques for JavaScript, which use more traditional static [Jensen et al. 2009; Thiemann 2005] or dynamic [Gong et al. 2015; Pradel et al. 2015] analysis. A recent survey gives a comprehensive overview of these techniques [Andreasen et al. 2017]. The main difference to this work is that DeepBugs exploits natural language information and machine learning to detect bugs.

\subsection{Other Related Work}

Our idea to create artificial, negative examples to train a binary classifier can be seen as a variant of noise-contrastive estimation [Gutmann and Hyvärinen 2010]. The novelty is to apply this approach to programs and to show that it yields effective bug detectors. The code transformations we use to 
create negative training examples relate to mutation operators [Jia and Harman 2011]. Additional mutation operators for name-related bugs, e.g., mined from version histories [Brown et al. 2017], could be an interesting way to extend DeepBugs with further bug detectors.

To evaluate bug detection tools, automated bug seeding has been proposed. LAVA [Dolan-Gavitt et al. 2016] and EvilCoder [Pewny and Holz 2016] introduce artificial security bugs into code to evaluate vulnerability detection tools. Their work differs from ours by focusing on vulnerabilities instead of name-related bugs and by seeding bugs for a different purpose.

\section{CONCLUSIONS}

This paper addresses the problem of name-based bug detection through a machine learning-based approach. Our DeepBugs framework learns bug detectors that distinguish correct from incorrect code by reasoning about the identifier names involved in the code. The key insights that enable the approach are (i) that reasoning about identifiers based on a learned, semantic representation of names is beneficial and (ii) that artificially seeding bugs through simple code transformations yields accurate bug detectors that are effective also for real-world bugs. In contrast to previous work on name-based bug detection, we target a dynamically typed language, where names convey information about the intended semantics of code, which is valuable in the absence of static types. Applying our framework and three bug detectors built on top of it to a large corpus of code shows that the bug detectors have an accuracy between $89 \%$ and $95 \%$, and that they detect 102 programming mistakes with a true positive rate of $68 \%$. In the long term, we envision our work to complement manually designed bug detectors by learning from existing code and by replacing some of the human effort required to create bug detectors with computational effort.

\section{ACKNOWLEDGMENTS}

This work was supported in part by the German Research Foundation within the Emmy Noether project ConcSys and the Perf4JS project, by the German Federal Ministry of Education and Research and by the Hessian Ministry of Science and the Arts within CRISP, by the Hessian LOEWE initiative within the Software-Factory 4.0 project, by NSF grants CCF-1409872 and CCF-1423645, and by a gift from Fujitsu Laboratories of America, Inc.

\section{REFERENCES}

Edward Aftandilian, Raluca Sauciuc, Siddharth Priya, and Sundaresan Krishnan. 2012. Building Useful Program Analysis Tools Using an Extensible Java Compiler. In 12th IEEE International Working Conference on Source Code Analysis and Manipulation, SCAM 2012, Riva del Garda, Italy, September 23-24, 2012. 14-23.

Miltiadis Allamanis, Earl T. Barr, Christian Bird, and Charles A. Sutton. 2014. Learning natural coding conventions. In Proceedings of the 22nd ACM SIGSOFT International Symposium on Foundations of Software Engineering, (FSE-22), Hong Kong, China, November 16 - 22, 2014. 281-293.

Miltiadis Allamanis, Earl T. Barr, Premkumar Devanbu, and Charles Sutton. 2017a. A Survey of Machine Learning for Big Code and Naturalness. arXiv:1709.06182 (2017).

Miltiadis Allamanis and Marc Brockschmidt. 2017. SmartPaste: Learning to Adapt Source Code. CoRR abs/1705.07867 (2017). http://arxiv.org/abs/1705.07867

Miltiadis Allamanis, Marc Brockschmidt, and Mahmoud Khademi. 2017b. Learning to Represent Programs with Graphs. CoRR abs/1711.00740 (2017). arXiv:1711.00740 http://arxiv.org/abs/1711.00740

Miltiadis Allamanis, Hao Peng, and Charles A. Sutton. 2016. A Convolutional Attention Network for Extreme Summarization of Source Code. In Proceedings of the 33nd International Conference on Machine Learning, ICML 2016, New York City, NY, USA, fune 19-24, 2016. 2091-2100.

Uri Alon, Meital Zilberstein, Omer Levy, and Eran Yahav. 2018. A General Path-Based Representation for Predicting Program Properties. In PLDI.

Glenn Ammons, Rastislav Bodík, and James R. Larus. 2002. Mining specifications. In Symposium on Principles of Programming Languages (POPL). ACM, 4-16. 
M. Amodio, S. Chaudhuri, and T. Reps. 2017. Neural Attribute Machines for Program Generation. ArXiv e-prints (May 2017). arXiv:cs.AI/1705.09231

Esben Andreasen, Liang Gong, Anders Møller, Michael Pradel, Marija Selakovic, Koushik Sen, and Cristian alexandru Staicu. 2017. A Survey of Dynamic Analysis and Test Generation for JavaScript. Comput. Surveys (2017).

Sahil Bhatia and Rishabh Singh. 2016. Automated Correction for Syntax Errors in Programming Assignments using Recurrent Neural Networks. CoRR abs/1603.06129 (2016).

Pavol Bielik, Veselin Raychev, and Martin T. Vechev. 2016. PHOG: Probabilistic Model for Code. In Proceedings of the 33nd International Conference on Machine Learning, ICML 2016, New York City, NY, USA, fune 19-24, 2016. 2933-2942.

David Bingham Brown, Michael Vaughn, Ben Liblit, and Thomas W. Reps. 2017. The care and feeding of wild-caught mutants. In Proceedings of the 2017 11th foint Meeting on Foundations of Software Engineering, ESEC/FSE 2017, Paderborn, Germany, September 4-8, 2017. 511-522.

Simon Butler, Michel Wermelinger, Yijun Yu, and Helen Sharp. 2010. Exploring the Influence of Identifier Names on Code Quality: An Empirical Study. In European Conference on Software Maintenance and Reengineering (CSMR). IEEE, 156-165.

Cristiano Calcagno, Dino Distefano, Jérémy Dubreil, Dominik Gabi, Pieter Hooimeijer, Martino Luca, Peter OâÁŹHearn, Irene Papakonstantinou, Jim Purbrick, and Dulma Rodriguez. 2015. Moving fast with software verification. In NASA Formal Methods Symposium. 3-11.

Daniel DeFreez, Aditya V. Thakur, and Cindy Rubio-González. 2018. Path-Based Function Embedding and its Application to Specification Mining. CoRR abs/1802.07779 (2018).

Brendan Dolan-Gavitt, Patrick Hulin, Engin Kirda, Tim Leek, Andrea Mambretti, William K. Robertson, Frederick Ulrich, and Ryan Whelan. 2016. LAVA: Large-Scale Automated Vulnerability Addition. In IEEE Symposium on Security and Privacy, SP 2016, San fose, CA, USA, May 22-26, 2016. 110-121.

ECMA. 2011. Standard ECMA-262, ECMAScript Language Specification, 5.1 Edition. (June 2011).

Dawson Engler, David Yu Chen, Seth Hallem, Andy Chou, and Benjamin Chelf. 2001. Bugs as Deviant Behavior: A General Approach to Inferring Errors in Systems Code. In Symposium on Operating Systems Principles (SOSP). ACM, 57-72.

Patrice Godefroid, Hila Peleg, and Rishabh Singh. 2017. Learn\&Fuzz: Machine Learning for Input Fuzzing. CoRR abs/1701.07232 (2017).

Liang Gong, Michael Pradel, and Koushik Sen. 2015. JITProf: Pinpointing JIT-unfriendly JavaScript Code. In European Software Engineering Conference and Symposium on the Foundations of Software Engineering (ESEC/FSE). 357-368.

Xiaodong Gu, Hongyu Zhang, Dongmei Zhang, and Sunghun Kim. 2016. Deep API learning. In Proceedings of the 24th ACM SIGSOFT International Symposium on Foundations of Software Engineering, FSE 2016, Seattle, WA, USA, November 13-18, 2016. 631-642. DOI : http://dx.doi.org/10.1145/2950290.2950334

Rahul Gupta, Soham Pal, Aditya Kanade, and Shirish Shevade. 2017. DeepFix: Fixing Common C Language Errors by Deep Learning. In $A A A I$.

Michael Gutmann and Aapo Hyvärinen. 2010. Noise-contrastive estimation: A new estimation principle for unnormalized statistical models. In Proceedings of the Thirteenth International Conference on Artificial Intelligence and Statistics. 297-304.

Andrew Habib and Michael Pradel. 2018. Is This Class Thread-Safe? Inferring Documentation using Graph-based Learning. In ASE. ASE.

Quinn Hanam, Fernando Santos De Mattos Brito, and Ali Mesbah. 2016. Discovering bug patterns in JavaScript. In Proceedings of the 24th ACM SIGSOFT International Symposium on Foundations of Software Engineering, FSE 2016, Seattle, WA, USA, November 13-18, 2016. 144-156. DOI : http://dx.doi.org/10.1145/2950290.2950308

Sudheendra Hangal and Monica S. Lam. 2002. Tracking down software bugs using automatic anomaly detection. In International Conference on Software Engineering (ICSE). ACM, 291-301.

Abram Hindle, Earl T. Barr, Zhendong Su, Mark Gabel, and Premkumar T. Devanbu. 2012. On the naturalness of software. In 34th International Conference on Software Engineering, ICSE 2012, fune 2-9, 2012, Zurich, Switzerland. 837-847.

Einar W. Høst and Bjarte M. Østvold. 2009. Debugging Method Names. In European Conference on Object-Oriented Programming (ECOOP). Springer, 294-317.

David Hovemeyer and William Pugh. 2004. Finding bugs is easy. In Companion to the Conference on Object-Oriented Programming, Systems, Languages, and Applications (OOPSLA). ACM, 132-136.

Min je Choi, Sehun Jeong, Hakjoo Oh, and Jaegul Choo. 2017. End-to-End Prediction of Buffer Overruns from Raw Source Code via Neural Memory Networks. CoRR abs/1703.02458 (2017).

Simon Holm Jensen, Anders Møller, and Peter Thiemann. 2009. Type Analysis for JavaScript. In Symposium on Static Analysis (SAS). Springer, 238-255.

Yue Jia and Mark Harman. 2011. An analysis and survey of the development of mutation testing. IEEE transactions on software engineering 37, 5 (2011), 649-678.

Dawn Lawrie, Christopher Morrell, Henry Feild, and David Binkley. 2006. What's in a Name? A Study of Identifiers. In International Conference on Program Comprehension (ICPC). IEEE, 3-12. 
Zhen Li, Shouhuai Xu Deqing Zou and, Xinyu Ou, Hai Jin, Sujuan Wang, Zhijun Deng, and Yuyi Zhong. 2018. VulDeePecker: A Deep Learning-Based System for Vulnerability Detection. In NDSS.

Bin Liang, Pan Bian, Yan Zhang, Wenchang Shi, Wei You, and Yan Cai. 2016. AntMiner: Mining More Bugs by Reducing Noise Interference. In ICSE.

Hui Liu, Qiurong Liu, Cristian-Alexandru Staicu, Michael Pradel, and Yue Luo. 2016. Nomen Est Omen: Exploring and Exploiting Similarities between Argument and Parameter Names. In International Conference on Software Engineering (ICSE). 1063-1073.

Peng Liu, Xiangyu Zhang, Marco Pistoia, Yunhui Zheng, Manoel Marques, and Lingfei Zeng. 2017. Automatic Text Input Generation for Mobile Testing. In ICSE.

Tomas Mikolov, Kai Chen, Greg Corrado, and Jeffrey Dean. 2013a. Efficient Estimation of Word Representations in Vector Space. CoRR abs/1301.3781 (2013). http://arxiv.org/abs/1301.3781

Tomas Mikolov, Ilya Sutskever, Kai Chen, Gregory S. Corrado, and Jeffrey Dean. 2013b. Distributed Representations of Words and Phrases and their Compositionality. In Advances in Neural Information Processing Systems 26: 27th Annual Conference on Neural Information Processing Systems 2013. Proceedings of a meeting held December 5-8, 2013, Lake Tahoe, Nevada, United States. 3111-3119.

Martin Monperrus, Marcel Bruch, and Mira Mezini. 2010. Detecting Missing Method Calls in Object-Oriented Software. In European Conference on Object-Oriented Programming (ECOOP). Springer, 2-25.

Lili Mou, Ge Li, Lu Zhang, Tao Wang, and Zhi Jin. 2016. Convolutional Neural Networks over Tree Structures for Programming Language Processing. In Proceedings of the Thirtieth AAAI Conference on Artificial Intelligence, February 12-17, 2016, Phoenix, Arizona, USA. 1287-1293.

Vijayaraghavan Murali, Swarat Chaudhuri, and Chris Jermaine. 2017. Finding Likely Errors with Bayesian Specifications. CoRR abs/1703.01370 (2017). http://arxiv.org/abs/1703.01370

Anh Tuan Nguyen and Tien N. Nguyen. 2015. Graph-Based Statistical Language Model for Code. In 37th IEEE/ACM International Conference on Software Engineering, ICSE 2015, Florence, Italy, May 16-24, 2015, Volume 1. 858-868.

Trong Duc Nguyen, Anh Tuan Nguyen, Hung Dang Phan, and Tien N. Nguyen. 2017. Exploring API embedding for API usages and applications. In Proceedings of the 39th International Conference on Software Engineering, ICSE 2017, Buenos Aires, Argentina, May 20-28, 2017. 438-449.

Jibesh Patra and Michael Pradel. 2016. Learning to Fuzz: Application-Independent Fuzz Testing with Probabilistic, Generative Models of Input Data. Technical Report TUD-CS-2016-14664. TU Darmstadt.

Jannik Pewny and Thorsten Holz. 2016. EvilCoder: automated bug insertion. In Annual Conference on Computer Security Applications (ACSAC). 214-225.

Michael Pradel and Thomas R. Gross. 2011. Detecting anomalies in the order of equally-typed method arguments. In International Symposium on Software Testing and Analysis (ISSTA). 232-242.

Michael Pradel, Ciera Jaspan, Jonathan Aldrich, and Thomas R. Gross. 2012. Statically Checking API Protocol Conformance with Mined Multi-Object Specifications. In International Conference on Software Engineering (ICSE). 925-935.

Michael Pradel, Parker Schuh, and Koushik Sen. 2015. TypeDevil: Dynamic Type Inconsistency Analysis for JavaScript. In International Conference on Software Engineering (ICSE).

Baishakhi Ray, Vincent Hellendoorn, Saheel Godhane, Zhaopeng Tu, Alberto Bacchelli, and Premkumar T. Devanbu. 2016. On the "naturalness" of buggy code. In Proceedings of the 38th International Conference on Software Engineering, ICSE 2016, Austin, TX, USA, May 14-22, 2016. 428-439.

Veselin Raychev, Pavol Bielik, and Martin Vechev. 2016a. Probabilistic Model for Code with Decision Trees. In OOPSLA.

Veselin Raychev, Pavol Bielik, Martin T. Vechev, and Andreas Krause. 2016b. Learning programs from noisy data. In Proceedings of the 43rd Annual ACM SIGPLAN-SIGACT Symposium on Principles of Programming Languages, POPL 2016, St. Petersburg, FL, USA, January 20 - 22, 2016. 761-774.

Veselin Raychev, Martin T. Vechev, and Andreas Krause. 2015. Predicting Program Properties from "Big Code".. In Principles of Programming Languages (POPL). 111-124.

Veselin Raychev, Martin T. Vechev, and Eran Yahav. 2014. Code completion with statistical language models. In ACM SIGPLAN Conference on Programming Language Design and Implementation, PLDI '14, Edinburgh, United Kingdom - June 09 - 11, 2014. 44.

Andrew Rice, Edward Aftandilian, Ciera Jaspan, Emily Johnston, Michael Pradel, and Yulissa Arroyo-Paredes. 2017. Detecting Argument Selection Defects. In OOPSLA.

Peter Thiemann. 2005. Towards a Type System for Analyzing JavaScript Programs. In European Symposium on Programming (ESOP). 408-422

Bogdan Vasilescu, Casey Casalnuovo, and Premkumar T. Devanbu. 2017. Recovering clear, natural identifiers from obfuscated JS names. In Proceedings of the 2017 11th foint Meeting on Foundations of Software Engineering, ESEC/FSE 2017, Paderborn, Germany, September 4-8, 2017. 683-693.

Proc. ACM Program. Lang., Vol. 2, No. OOPSLA, Article 147. Publication date: November 2018. 
Song Wang, Taiyue Liu, and Lin Tan. 2016. Automatically learning semantic features for defect prediction. In Proceedings of the 38th International Conference on Software Engineering, ICSE 2016, Austin, TX, USA, May 14-22, 2016. 297-308.

Andrzej Wasylkowski and Andreas Zeller. 2009. Mining Temporal Specifications from Object Usage. In International Conference on Automated Software Engineering (ASE). IEEE, 295-306.

Jason Weston, Sumit Chopra, and Antoine Bordes. 2014. Memory Networks. CoRR abs/1410.3916 (2014).

Martin White, Michele Tufano, Christopher Vendome, and Denys Poshyvanyk. 2016. Deep learning code fragments for code clone detection. In Proceedings of the 31st IEEE/ACM International Conference on Automated Software Engineering, ASE 2016, Singapore, September 3-7, 2016. 87-98.

Xin Ye, Hui Shen, Xiao Ma, Razvan C. Bunescu, and Chang Liu. 2016. From word embeddings to document similarities for improved information retrieval in software engineering. In Proceedings of the 38th International Conference on Software Engineering, ICSE 2016, Austin, TX, USA, May 14-22, 2016. 404-415. 